\title{
Endoscopic characteristics and causes of misdiagnosis of intestinal schistosomiasis
}

\author{
CHUNCUI YE, SHIYUN TAN, LIN JIANG, MING LI, PENG SUN, LEI SHEN and HESHENG LUO \\ Department of Gastroenterology, Renmin Hospital of Wuhan University, Wuhan, Hubei 430060, P.R. China
}

Received February 21, 2013; Accepted July 27, 2013

DOI: $10.3892 / \mathrm{mmr} .2013 .1648$

\begin{abstract}
The aim of this study was to determine the clinical and endoscopic manifestations, and pathological characteristics of intestinal schistosomiasis in China, in order to raise awareness of intestinal schistosomiasis and prevent misdiagnosis and missed diagnosis. The retrospective analysis of clinical and endoscopic manifestations, and histopathological characteristics, were conducted for 96 patients with intestinal schistosomiasis. Among these patients, 21 lived in areas that were not infected with Schistosoma and 25 (26\%) had no history of schistosome infection or contact with infected water. These patients were mainly hospitalized due to symptoms of diarrhea, mucus and bloody purulent stool. Sixteen cases were of the acute enteritis type, and colonoscopy results determined hyperaemic edema and dispersed small mucosal ulcers. The acute infection in patients was pathologically characterized by the deposition of intact ova with a large quantity of eosinocyte infiltration. Forty-one cases were of the chronic enteritis type which predominantly manifested with yellow nodules and disorder of the vascular surfaces in the intestines. Thirty-nine cases were diagnosed with mixed type enteritis, which demonstrated acute and chronic histopathological appearances. In addition, six cases of complicated colorectal cancer were observed. Of the 24 misdiagnosed patients, eight were misdiagnosed with ulcerative colitis, five with colorectal cancer, five with colorectal tuberculosis, four with chronic bacillary dysentery and two with irritable bowel syndrome. Intestinal schistosomiasis demonstrated no specific clinical or endoscopic manifestations and it was determined that patients with abdominal pain, diarrhea and mucous stool may be infected with intestinal schistosomiasis. Epidemiological investigations and colonoscopy combined with multi-block and multi-site biopsies may improve the diagnosis of intestinal schistosomiasis. In addition, it is necessary for intestinal schistosomiasis to be followed up by colonoscopy, due to its possible correlation with colorectal tumors.
\end{abstract}

Correspondence to: Professor Shiyun Tan, Department of Gastroenterology, Renmin Hospital of Wuhan University, 238 Republic Road, Wuhan, Hubei 430060, P.R. China

E-mail: tanshiyun@medmail.com.cn

Key words: schistosomiasis, colonoscopy, colorectal carcinoma, misdiagnosis

\section{Introduction}

Schistosomiasis is one of the most widespread parasitic infections worldwide. The three predominant schistosome species identified to infect humans are Schistosoma haematobium (endemic in Africa and the eastern Mediterranean), S. mansoni (endemic in Africa, the Middle East, the Caribbean and South America) and S. japonicum (endemic mainly in China, Japan and the Philippines) (1). It is estimated that 843,007 individuals in China are infected with $S$. japonicum (2). Intestinal schistosomiasis is an acute or chronic, specific enteropathy caused by the deposition of schistosome ovum on the colon and rectal walls. S. japonicum is prevalent in China and, following efforts to control schistosome infection, the incidence of acute schistosome infection has decreased to only a few sporadic cases (3-8). The intestinal and general symptoms of schistosome infection are not typical (9), thus, the accurate rate of diagnosis is low and the infection may be easily misdiagnosed.

Adult S. japonicum worms live in the mesenteric veins in pairs and lay numerous eggs in the gastrointestinal mucosa ( $\sim 3000 /$ day/pair). The eggs are responsible for inflammatory and immunopathological responses, leading to erythema, edema, granuloma formation, ulceration, hemorrhage and fibrosis. Gastrointestinal symptoms may include nausea, meteorism, abdominal pain, bloody diarrhea, rectal tenesmus and hepatosplenomegaly (10). In addition, the clinical manifestations are correlated with the severity of the infection. In order to prevent the misdiagnosis of schistosoma infection, a rational approach to the endoscopic diagnosis is required.

In the present study, we retrospectively analyzed the clinical, endoscopic and pathological features of 96 patients with intestinal schistosomiasis, which were diagnosed by a combination of endoscopic biopsy and histopathological examination from June 2000 to June 2010, at the Renmin Hospital of Wuhan University (Wuhan, Hubei, China).

\section{Patients and methods}

Patients. We studied 96 patients diagnosed among 37,865 patients with intact endoscopic and histopathology records at the Department of Gastroenterology (Renmin Hospital of Wuhan University) between June 2000 and June 2010. There were 72 male and 24 female patients aged 16-79 years (average age, 44.7 years). The clinical time since the symptoms appeared ranged from 2 months to 25 years. The study was approved by 
the Ethics Committee of Renmin Hospital of Wuhan University, Wuhan, China and written informed consent was obtained from the patients/patients' families.

Collecting clinical data. The medical history, clinical symptoms, colonoscopy manifestation and pathological data of the patients with intestinal schistosomiasis were obtained from the Digestive Endoscopy Center and Record Room of Renmin Hospital.

Endoscopy. The CF240 (Olympus, Tokyo, Japan) or the CF260 (Fujifilm, Tokyo, Japan) electronic colonoscope was used to examine the patients. During the colonoscopy examination, at least three colonic mucosal biopsies were taken, and normal colonic mucosa and polyps were removed using high frequency electrocoagulation and electrocision. Pathological tissues from the biopsies were sent for histopathological examination at the Department of Pathology, Renmin Hospital of Wuhan University.

Histopathology. The specimens for histopathological examination were fixed in $10 \%$ formaldehyde, dehydrated with various concentrations of alcohol and xylol, then paraffin-embedded and cut into $4-\mu \mathrm{m}$ sections. Hematoxylin and eosin staining was used to observe the pathological changes under a light microscope (Olympus, Tokyo, Japan).

\section{Results}

Patient history and clinical symptoms. Among 96 patients with intestinal schistosomiasis, 57 had received anthelmintic treatment, 21 lived in areas without schistosome infection, and 25 (26\%) had no history of schistosome infection and no apparent contact with infested water. The patients were mainly hospitalized for abdominal pain, diarrhea, mucus and bloody purulent stool, with stool passage two to seven times per day. Of the 96 intestinal schistosomiasis patients, there were 46 with a low fever, emaciation and asthenia; 17 with abdominal masses; and 61 with a degree of splenomegaly (Table I).

Position of lesions. Of the 96 patients in this study, 92 were analyzed by colonoscopy, with the colonoscope inserted to the end of the ileum, to determine the location of the pathological changes (Table II).

Colonoscopic manifestations. Generally, intestinal schistosomiasis was divided into three types: Acute colonitis, chronic colonitis and mixed colonitis (11), as demonstrated in Table III

Sixteen cases of acute colonitis were observed with endoscopy and exhibited hyperaemic edema, mucus exudation, vague vascular striation and scattered aphtha and punctate hemorrhage of the intestinal mucosae (Fig. 1). Among 16 patients, nine had typical yellow nodules that were commonly distributed around severe small ulcers.

Among the 96 patients, there were 41 chronic colonitis cases (Figs. 2 and 3), in which the intestinal mucosae presented with mucosal thickening, loss of vascular texture, paleness, cicatrization, haustrum blockages and polypoid protrusion. In addition, in a small number of cases, scattered cankers were observed. Twenty-six patients demonstrated merged colon polyps, and
Table I. Clinical symptoms of 96 cases of intestinal schistosomiasis.

\begin{tabular}{lc}
\hline Clinical symptom & $\begin{array}{r}\text { No. of cases } \\
(\mathrm{n}=96)\end{array}$ \\
\hline Diarrhea & 64 \\
Mucous stool & 47 \\
Abdominal distention & 39 \\
Abdominal pain & 75 \\
Low fever, emaciation and asthenia & 46 \\
Splenomegaly & 61 \\
Bloody purulent stool & 21 \\
Constipation & 23 \\
Alternation of diarrhea & 9 \\
and constipation & \\
Abdominal mass & 17 \\
Intestinal obstruction & 2 \\
\hline
\end{tabular}

Table II. Location of pathological change.

\begin{tabular}{lc}
\hline Location of pathological change & $\begin{array}{c}\text { No. of cases } \\
(\mathrm{n}=96)\end{array}$ \\
\hline Rectum & 47 \\
Sigmoid colon & 55 \\
Descending colon & 11 \\
Transverse colon & 8 \\
Ascending colon & 6 \\
Cecum & 9 \\
\hline
\end{tabular}

39 patients had typical yellow nodules which were of a more concentrated distribution compared with those of the acute type.

There were 39 cases of mixed colonitis, in which the intestinal mucosae demonstrated the combined manifestations of the aforementioned two types of colonitis. A combination of acute (primarily in the left intestines) and chronic (primarily in the right intestines) inflammation occurred in certain sections. In addition, the two types of inflammation were sometimes observed alongside each other, thus rendering it difficult to distinguish between primary infection and infection recurrence. Twenty-eight patients had scattered yellow nodules and 14 had merged colon polyps.

There were six cases of intestinal schistosomiasis combined with colorectal cancer (Fig. 2), two cases of which were located in the sigmoid colon and rectum, and four of which were located in the ileocecal valve. Under endoscopy, two cases were observed with the formation of neoplasma and four manifested with stenosis.

Histopathological characteristics. Schistosome ovum, deposited in the lamina propria, were detected in the endoscopic biopsies of all 96 patients. The 16 patients with acute colonitis pathologically manifested with the infiltration of numerous interstitial eosinocytes and neutrophilic granulocytes, and 
Table III. Colonoscopy manifestations of intestinal schistosomiasis.

\begin{tabular}{lccc}
\hline Colonoscopy manifestation & Acute colonitis $(\mathrm{n}=16)$ & Chronic colonitis $(\mathrm{n}=41)$ & Mixed colonitis $(\mathrm{n}=39)$ \\
\hline Hyperaemic edema & 16 & 6 & 10 \\
Mucus exudation & 15 & 5 & 15 \\
Anabiosis and canker & 10 & 13 & 4 \\
Yellow nodules & 9 & 39 & 28 \\
Disorder of vascular textures & 0 & 41 & 30 \\
Cicatrice & 0 & 32 & 23 \\
Polypus & 0 & 26 & 14 \\
Neoplasm & 0 & 1 & 1 \\
Stenosis & 0 & 4 & 0 \\
\hline
\end{tabular}

uncalcified schistosome ovum deposition in the lamina propria under the mucosa (Fig. 4). The 41 patients with chronic colonitis predominantly demonstrated lymphocyte and plasmocytes at the submucosa and lamina propria. Atrophy of the intestinal mucosal epithelium, reduction of the intestinal gland, hyperplasia of partial submucosa tissue accompanied with fibrosis and chronic ova nodules were also observed, however several calcified schistosome ovum were hidden (Fig. 5). There were 39 cases of mixed colonitis with combined manifestations of acute and chronic enteritis. Among which, certain mucosal segments demonstrated an infiltration of lymphocytes while others demonstrated an infiltration of eosinocytes.

Forty-one cases were complicated with colorectal polyps, all of which were removed with high frequency electrocoagulation and electrocision. Patholgical analysis demonstrated that these were hyperplastic or inflammatory polyps. In addition, no polyps were observed in acute colorectal enteritis. Six out of 96 intestinal schistosomiasis patients also demonstrated the presence of colorectal cancer. Two cases of mucinous adenocarcinoma, two mid-differentiated tubular adenocarcinoma, one papillary adenocarcinoma and one mixed carcinoma were detected. The deposition of schistosome ovum and tumor cells was examined simultaneously in the same pathological specimen in six patients (Fig. 6). A medium or heavy degree of hyperplasia considered as precancerosis was observed in nine cases.

Misdiagnosis. In the group of 96 cases, 24 were misdiagnosed outside the hospital (misdiagnosis rate, 25\%). Eight of the 24 cases were misdiagnosed as ulcerative colitis, five as ileocecal tumor, five as intestinal tuberculosis, four as chronic bacillary dysentery and two as irritable bowel syndrome.

Endoscopic features of the intestinal mucosa in the 24 misdiagnosed cases were non-typical. Eight cases demonstrated intensive or scattered ulcer-like changes with varying size and depth. The majority of the ulcers were congestive and edematous and attached by secretions to the peripheral mucosa. In addition, the typical yellow nodules in the intestinal tract were rare; thus, the cases were misdiagnosed as ulcerative colitis. Five cases exhibited the deformation of the ileocecal valve, occasionally accompanied by scattered non-typical yellow nodules and friable mucosa with the increased likelihood of contact bleeding. As a result, they were misdiagnosed as ileocecal tumors. Another five cases, with lesions in the sigmoid colon demonstrated granular protrusions of the intestinal mucosa, small scattered ulcers, intestinal stiffness, narrowed lumen and stenosis, combined with a low-level fever in patients. These cases were misdiagnosed as intestinal tuberculosis. Furthermore, biopsies were not taken in six out of 24 cases, while in another 18 cases, an insufficiently sized specimen was collected; thus, in these cases, the ova nodules were not located.

\section{Discussion}

It is generally acknowledged that the endoscopic features of intestinal schistosomiasis may be divided into the acute colitis type and the chronic colitis type $(11,12)$. The mucosas of the acute colitis type predominantly expressed congestion and edema. By contrast, the chronic colitis type expressed chronic inflammation, such as mucosal hypertrophy and scarring. The ova nodules of the acute colitis type were acute and consisted of one or multiple central ova surrounded by eosinocytes. By contrast, in the chronic colitis type, the middle ova of the nodules had ruptured and calcified, and were surrounded by lymphocytes and epithelioid cells. This was accompanied by the proliferation of numerous submucosal fibrous tissues. In the 96 patients, 16 were of the acute colitis type and 41 were of the chronic colitis type; however, 39 demonstrated a combination of acute and chronic mucosal inflammation changes and the biopsies showed the concurrence of 2 types of ova nodules. This is consistent with the results of a study by Liu et al (13) in which adding the mixed type to the classification of shistosomiasis was proposed.

Recently, the morbidity of intestinal schistosomiasis has increased due to inadequate knowledge. Numerous patients in affected areas suffer from chronic schistosomiasis. Due to the lack of thorough treatment and repeated infection, these patients demonstrated a combination of chronically inflamed pathological manifestations with acute inflammation. As a result of the multiple and atypical clinical manifestations of these patients, misdiagnosis was common. For example, patients with acute inflammatory manifestations, such as fever, abdominal pain, diarrhea and bloody stools, were often misdiagnosed with bacillary or amebic dysentery. Additionally, those exhibiting chronic inflammation, such as abdominal pain and diarrhea, were often misdiagnosed with ulcerative colitis, intestinal tuberculosis and colon cancer. Hence, it is essential for clinicians to consider 


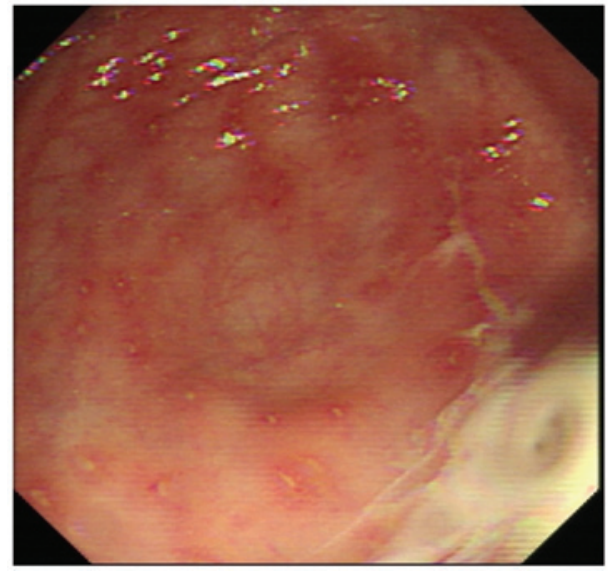

Figure 1. Endoscopic manifestation of acute intestinal schistosomiasis: Mucosal hyperemia and edema, mucus exudation, vague vascular striation and scattered small ulcers and yellow nodules.

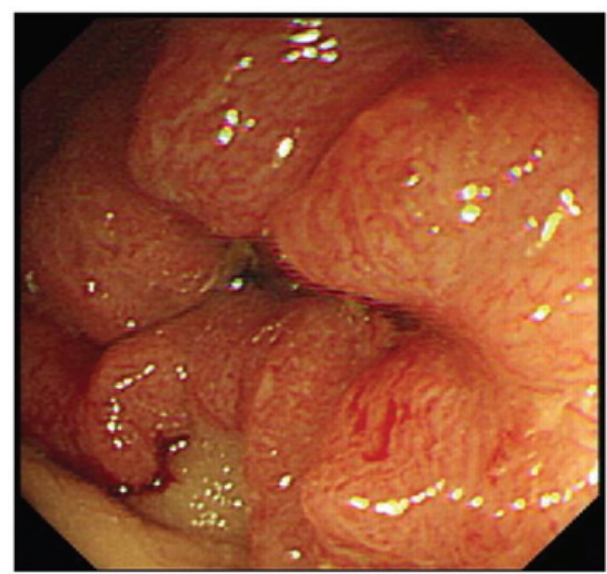

Figure 2. Endoscopic manifestation of chronic intestinal schistosomiasis. The colon mucosae present with hyperaemic edema and stenosis of the colon cavity combined with with colon cancer.

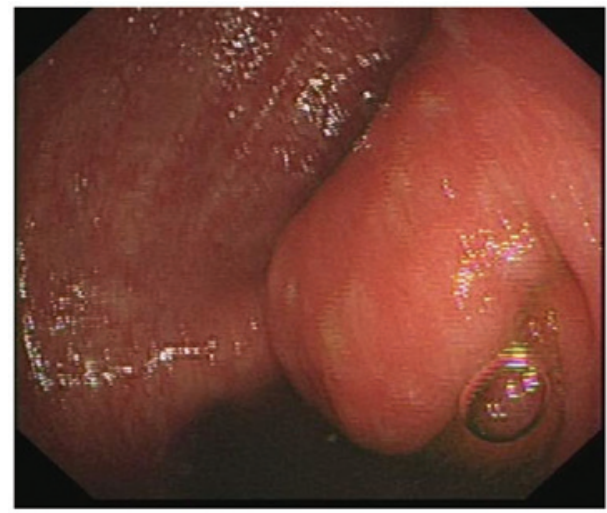

Figure 3. Endoscopic manifestation of chronic intestinal schistosomiasis, mucosal thickening and cicatrize with polypoid protrusion.

schistosomiasis when making a diagnosis, particularly in patients living in or in contact with infected areas.

Among 96 cases of intestinal schistosomiasis, 24 cases were misdiagnosed outside the hospital and were examined at the Renmin Hospital due to the lack of effective treatment.

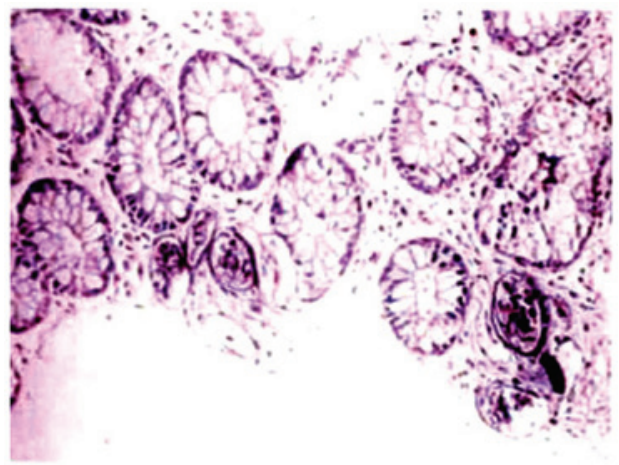

Figure 4. Histopathological manifestation of acute intestinal schistosomiasis, acute ova nodules, intact ova with numerous eosinophils infiltrating. Staining, hematoxylin and eosin; magnification, $\mathrm{x} 20$.

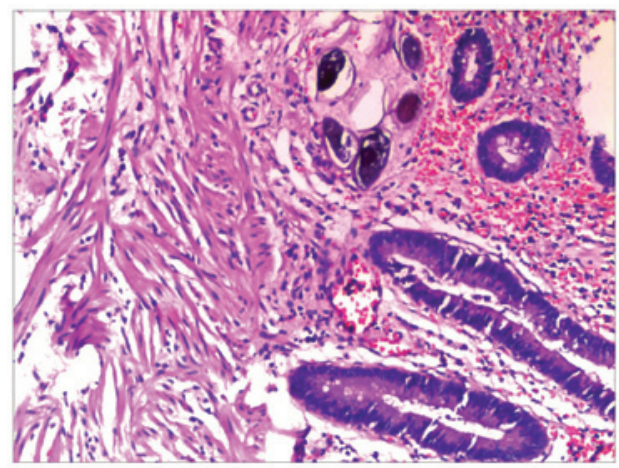

Figure 5. Histopathological manifestations of chronic intestinal schistosomiasis with tubular adenoma, calcified schistosome ovum, lymphocyte and plasmocyte infiltration, and proliferation of submucosal tissues. Staining, hematoxylin and eosin; magnification, $\mathrm{x} 40$.

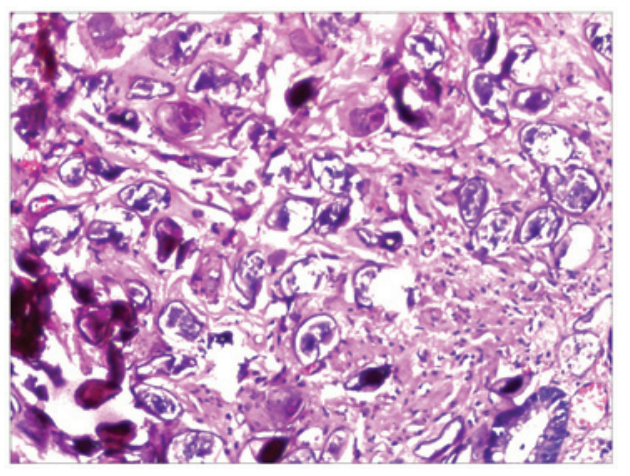

Figure 6. Histopathological manifestation of chronic intestinal schistosomiasis, chronic ova nodules and calcified ova in carcinoma foci. Staining, hematoxylin and eosin; magnification x40.

The causes of misdiagnosis were demonstrated to be partly due to the diverse and complex endoscopic features of these patients and also to the mucosal biopsies. In certain patients, this was due to the fact that biopsies had not been conducted; however, for the majority, the biopsies were derived from inaccurate positions or samples were insufficient in size. The lack of effective biopsies meant that the egg depositions had not been located and thus resulted in misdiagnosis.

The endoscopic manifestations of intestinal schistosomiasis are prone to be confused with those of ulcerative colitis, 
Crohn's disease, intestinal tuberculosis and colon cancer without careful identification. For example, the intestinal mucosas of the acute colitis type of intestinal schistosomiasis manifest as congestion, edema and ulcers; while those of the chronic colitis type exhibit mucosal hypertrophy, granuloma or polyp formation and a narrowed, hardened lumen, which may be misdiagnosed as active or recovery ulcerative colitis, or intestinal tuberculosis. The presence of granular hyperplasia, multiple nodules or large granuloma and large ulcers in the intestine lumen may lead to a misdiagnosis of colon cancer.

Although the incidence of intestinal schistosomiasis has decreased compared with that in the 1970s and 1980s, it has not been completely eliminated. Therefore, it is important to determine patients' history of exposure to infected water. Moreover, it is necessary to be aware of the endoscopic features of intestinal schistosomiasis and to carefully distinguish these from those of ulcerative colitis, intestinal tuberculosis, intestinal cancer and other diseases. In addition, it is also necessary to analyze biopsies, particularly the multi-site, multi-block biopsies for suspected cases. This is due to the fact that, when the typical histopathological features of intestinal schistosomiasis were observed, it was diagnosed. The results were concordant with a study by Ohmae et al in which the schistosome egg nodules observed in histological specimens were proposed to be the gold standard for the diagnosis of this disease (14).

It is suggested that intestinal schistosomiasis is a risk factor for colorectal cancer; however, this remains controversial. Clinical pathological studies have demonstrated that the concurrent incidence of intestinal schistosomiasis and colorectal cancer was higher than that of the control group $(15,16)$. In the present study, of the 96 patients with intestinal schistosomiasis, six cases were accompanied by colorectal cancer and nine with a medium or heavy degree of hyperplasia considered to be precancerosis. According to the results, it was determined that intestinal schistosomiasis may be correlated with the occurrence of colorectal cancer.

A possible mechanism for the induction of cancer by intestinal schistosomiasis may be the deposition of schistosome eggs in the digestive tract and the accumulation of a large number of eggs under the intestinal mucosa, which may lead to chronic mucosal inflammation, thus resulting in polypoid hyperplasia and canceration. Li et al (17) observed that deposited schistosome ova are important in the carcinogenesis of colorectal cancer. It was also demonstrated that colorectal cancer foci were often located in significant sections of intestinal schistosomiasis; the possible factors were the presence of endogenously produced carcinogens (18), chronic immunomodulation resulting in impairment of immunological surveillance (19), symbiotic action of other infective agents (20) and the presence of schistosomal toxins (21). It is possible that the intestinal mucosa is constantly stimulated by schistosome eggs, which leads to mutations in genes of the mucosal gland epithelial cells (15). Thus, investigation at the molecular level is required to determine the precise correlation between intestinal schistosomiasis and colorectal cancer.

In conclusion, intestinal schistosomiasis is risk factor for colorectal cancer and the lesions of the disease may be considered as precancerous. Therefore, histopathological analysis of periodic endoscopic follow-up and multi-block and multi-site endoscopic biopsy are important. To reduce the incidence of colorectal cancer, early endoscopic or surgical treatment for patients with schistosome infection combined with colon polyps is required.

\section{Acknowledgements}

This study was supported by the Health Department Foundation of Hubei Province, China (grant no. XF2010-21).

\section{References}

1. Gryseels B, Polman K, Clerinx J and Kestens L: Human schistosomiasis. Lancet 368: 1106-1118, 2006.

2. Zhou XN, Wang TP, Wang LY et al: The current status of schistosomiasis epidemics in China. Zhonghua Liu Xing Bing Xue Za Zhi 25: 555-558, 2004 (In Chinese).

3. Wang W, Dai JR, Liang YS, Huang YX and Coles GC: Impact of the South-to-North Water Diversion Project on the transmission of Schistosoma japonicum in China. Ann Trop Med Parasitol 103: 17-29, 2009.

4. Wu GY and Halim MH: Schistosomiasis: progress and problems. World J Gastroenterol 6: 12-19, 2000.

5. Zhou X: Distribution and control of the schistosomiasis in China. Journal of Anhui Agri Sci 12: 3766-3768, 2007 (In Chinese).

6. Zhang LJ, Zhu R, Dang H, Xu J, Li SZ and Guo JG: Analysis of surveillance of schistosomiasis in China in 2011. Zhongguo Xue Xi Chong Bing Fang Zhi Za Zhi 24: 627-631, 2012 (In Chinese).

7. Wang Y, Wang YR, Ying XJ and Zhu XC: Epidemic situation of schistosomiasis in a provincial surveillance site of Shengzhou City, 2008-2011. Zhongguo Xue Xi Chong Bing Fang Zhi Za Zhi 25: 104-105, 2013 (In Chinese).

8. Sun LP, Tian ZX, Yang K, Huang YX, et al: Effect evaluation of transmission control of schistosomiasis in 14 counties (cities, districts) of Jiangsu Province. Zhongguo Xue Xi Chong Bing Fang Zhi Za Zhi 24: 26-31, 2012 (In Chinese).

9. Rizzo M, Mansueto P, Cabibi D, et al: A case of bowel schistosomiasis not adhering to endoscopic findings. World J Gastroenterol 11: 7044-7047, 2005.

10. Schafer TW and Hale BR: Gastrointestinal complications of schistosomiasis. Curr Gastroenterol Rep 3: 293-303, 2001.

11. Anthony BJ, Ramm GA and McManus DP: Role of resident liver cells in the pathogenesis of schistosomiasis. Trends Parasitol 28: 572-579, 2012.

12. Larkin BM, Smith PM, Ponichtera HE, Shainheit MG, Rutitzky LI and Stadecker MJ: Induction and regulation of pathogenic Th17 cell responses in schistosomiasis. Semin Immunopathol 34: 873-888, 2012.

13. Liu H, Liu L and Lu J: Diagnosis and Classification of Schistosomiasis in Children (report of 25 cases). Zhonghua xiao hua nei jing za zhi 16: 216-217, 1999 (In Chinese).

14. Ohmae H, Sy OS, Chigusa Y and Portillo GP: Imaging diagnosis of Schistosomiasis japonica - the use in Japan and application for field study in the present endemic area. Parasitol Int 52: 385-393, 2003.

15. Mei J, Hong H, Ding Y and Fang Y: Clinic pathological characteristics of chronic schistosomiasis concurrent colorectal Cancer. Chin J Dig Endosc 1: 49-50, 2004 (In Chinese).

16. Matsuda K, Masaki T, Ishii S, et al: Possible associations of rectal carcinoma with Schistosoma japonicum infection and membranous nephropathy: a case report with a review. Jpn J Clin Oncol 29: 576-581, 1999.

17. Li WC, Pan ZG and Sun YH: Sigmoid colonic carcinoma associated with deposited ova of Schistosoma japonicum: a case report. World J Gastroenterol 12: 6077-6079, 2006.

18. Rosin MP, Anwar WA and Ward AJ: Inflammation, chromosomal instability, and cancer: the schistosomiasis model. Cancer Res 54 (7 Suppl): 1929s-1933s, 1994

19. van Riet E, Hartgers FC, Yazdanbakhsh M: Chronic helminth infections induce immunomodulation: consequences and mechanisms. Immunobiology 212: 475-490, 2007.

20. Shindo K: Significance of schistosomiasis japonica in the development of cancer of the large intestine: Report of a case and review of the literature. Dis Colon Rectum 19: 460-469, 1976.

21. Long XC, Bahgat M, Chlichlia K, Ruppel A and Li YL: Detection of inducible nitric oxide synthase in Schistosoma japonicum and S. mansoni. J Helminthol 78: 47-50, 2004. 